\title{
Zero-dispersion stochastic resonance in a model for a superconducting quantum interference device
}

\author{
I. Kh. Kaufman, ${ }^{*}$ D. G. Luchinsky, ${ }^{*}$ P. V. E. McClintock, S. M. Soskin, ${ }^{\dagger}$ and N. D. Stein \\ School of Physics and Chemistry, Lancaster University, Lancaster LA1 4YB, United Kingdom
}

(Received 26 June 1997)

\begin{abstract}
It is demonstrated that the signal-to-noise ratio for a weak periodic signal in a superconductive loop with a Josephson junction (a superconducting quantum interference device, or SQUID) can be substantially enhanced, over a wide range of frequencies, by the addition of noise. This manifestation of zero-dispersion stochastic resonance (ZDSR) is shown to occur for a wide variety of loop parameters and signal frequencies. Unlike most earlier examples of stochastic resonance, ZDSR does not depend on fluctuational transitions between coexisting stable states. Rather, it exploits the noise-enhanced susceptibility that arises in underdamped nonlinear oscillators for which the oscillation eigenfrequency possesses one or more extrema as a function of energy. The phenomenon is investigated theoretically, and by means of analog and digital simulations. It is suggested that ZDSR could be used to enhance the sensitivity of radio-frequency SQUIDs and other SQUID-based devices. In the course of the work, two additional useful results were obtained: (a) an asymptotic expression describing ZDSR for the general case in the limit of weak dissipation; (b) a method for the numerical calculation of fluctuation spectra in bistable or multistable underdamped systems. [S1063-651X(97)08112-9]
\end{abstract}

PACS number(s): 05.40. $+\mathrm{j}$, 85.25.Dq, 03.20. $+\mathrm{i}, 05.45 .+\mathrm{b}$

\section{INTRODUCTION}

Stochastic resonance (SR) has been the subject of intensive investigation over the last decade: for recent reviews see $[1,2]$. The idea of SR was originally introduced $[3,4]$ in relation to a noise-induced enhancement of the amplitude of a low frequency periodic signal in a bistable system. It was subsequently realized $[5,6]$, however, that a stronger definition of SR in the same system was also possible: it was shown that, for small enough signals, not only the signal amplitude but also the signal-to-noise ratio (SNR) could increase with noise intensity (temperature) within a certain range. It is this latter definition of SR that is probably now the more widely used and accepted, and which we will apply below.

A general theory of SR, not confined to the conventional bistable case [6], was introduced in [7]. It was predicted [8,9] on this basis that SR could also occur in monostable systems [10]. In this perception of the phenomenon, SR is to be anticipated in any system whose fluctuation spectrum in the absence of a periodic signal displays at least one narrow peak that grows quickly enough with increasing noise intensity. In the particular case considered in [8,9], the SR was associated with zero-dispersion peaks [12-15] in systems whose eigenfrequency as a function of energy possesses an extremum: to distinguish it from conventional bistable SR, it was named zero-dispersion stochastic resonance (ZDSR).

Unfortunately, the model analyzed in $[8,9]$, the tilted single-well Duffing oscillator, typically requires such very small values of the damping parameter that SR (in terms of

\footnotetext{
*Permanent address: Research Institute for Metrological Service, Ozernaya 46, 119361 Moscow, Russia.

${ }^{\dagger}$ Permanent address: Institute of Semiconductor Physics, Ukrainian National Academy of Sciences, Kiev, Ukraine.
}

the strong definition above) could not be demonstrated in the analog electronic experiments [9]. It was noted in [15], however, that a model superconducting quantum interference device (SQUID) appeared to be much more promising, in this respect, because ZDSR was to be anticipated for considerably larger damping values. Our recent investigations [16] have confirmed this hypothesis, both experimentally and theoretically. In the present work, not only has the general theory of ZDSR been developed in detail, but we have also been able to investigate some interesting features of ZDSR that are peculiar to SQUIDs. The results could be of interest in terms of applications, given that SQUIDs are frequently used in practical devices [17]. It will become apparent that ZDSR can in principle be used to enhance the output SNR of a SQUID at moderate and high frequencies, in very much the same way as conventional SR has already been used to enhance the output SNR of multistable SQUIDs [18] in the low-frequency range.

The structure of the paper is as follows. General expressions for the SNR in terms of the fluctuation spectrum, and the asymptotic theory of ZDSR, are presented in Sec. II. The general theory of fluctuation spectra for underdamped motion is developed in Sec. III. Analog electronic experiments on a SQUID model, and their results, are described in Sec. IV. In Sec. V they are compared with the theory and discussed. The work is summarized and conclusions drawn in Sec. VI.

\section{THEORY OF ZERO-DISPERSION STOCHASTIC RESONANCE}

Let us consider a one-dimensional oscillator subject to a weak periodic force, and to friction and noise which, for the sake of convenience, we take to be linear and white, respectively, 


$$
\begin{gathered}
\ddot{q}+\Gamma \dot{q}+\frac{d U}{d q}=f(t)+A \cos (\Omega t), \\
\langle f(t)\rangle=0, \quad\left\langle f(t) f\left(t^{\prime}\right)\right\rangle=2 \Gamma T \delta\left(t-t^{\prime}\right),
\end{gathered}
$$

where $U(q)$ is a potential, and the noise intensity $T$ corresponds to temperature in cases where the noise is of thermal origin. We introduce the SNR [6] in terms of the power spectrum,

$$
Q(\omega)=\lim _{\tau \rightarrow \infty}(4 \pi \tau)^{-1}\left|\int_{-\tau}^{\tau} d t q(t) \exp (i \omega t)\right|^{2}
$$

$Q(\omega)$ consists of $\delta$ spikes at the frequency $\Omega$ of the periodic force, and its harmonics, superimposed on a smooth fluctuational background that corresponds to the power spectrum $Q^{(0)}(\omega)$ in the absence of the periodic force. The SNR is then defined as the ratio of the intensity (square) of the $\delta$ spike at $\Omega$ to the fluctuational background at $\Omega$ [6], so that

$$
\mathcal{R}=\frac{I_{\delta}(\Omega)}{Q^{(0)}(\Omega)} .
$$

As shown in [7], the $\mathrm{SNR}(\mathcal{R})$ can be written in terms of the complex susceptibility which, in turn, can be expressed in terms of $Q^{(0)}(\Omega)$ using the fluctuation dissipation theorem and the Kramers-Kronig relations

$$
\begin{gathered}
\mathcal{R}=\frac{1}{4} A^{2}|\chi(\Omega)|^{2} / Q^{(0)}(\Omega), \\
\operatorname{Re}[\chi(\Omega)]=\frac{2}{T} P \int_{0}^{\infty} d \alpha\left(\frac{\alpha^{2}}{\alpha^{2}-\Omega^{2}}\right) Q^{(0)}(\alpha), \\
\operatorname{Im}[\chi(\Omega)]=\frac{\pi \Omega}{T} Q^{(0)}(\Omega),
\end{gathered}
$$

where $\mathrm{P}$ denotes the Cauchy principal part and

$$
\begin{aligned}
Q^{(0)}(\Omega) & =\frac{1}{\pi} \operatorname{Re} \int_{0}^{\infty} d t \exp (-i \Omega t) \widetilde{Q}(t), \\
\widetilde{Q}(t) & =\langle[q(t)-\langle q\rangle][q(0)-\langle q\rangle]\rangle
\end{aligned}
$$

is the power spectrum in the absence of the periodic force.

It was shown in [8] that the SNR can increase with $T$ if $Q^{(0)}(\Omega)$ has a tall narrow peak that rises rapidly with $T$ and if the frequency $\Omega$ of the driving force lies within the range of the peak. Just such a situation can be realized for underdamped oscillators for which the dependence of an eigenoscillation frequency on energy possesses an extremum. The derivative of the eigenfrequency with respect to energy, $d \omega(E) / d E$ (the "dispersion" of the eigenfrequency), is equal to zero at the extremum. Correspondingly, energy fluctuations near the extremal energy, $E_{m}$, affect the phase much less than in other energy ranges, so that the correlation of the phase (and of the coordinate oscillations as well) lasts much longer. Consequently, $Q^{(0)}(\Omega)$ exhibits a tall narrow peak in the vicinity of the extremal eigenfrequency $\omega_{m} \equiv \omega\left(E_{m}\right)$, the so-called zero-dispersion peak (ZDP) [12,13] which, for small enough $\Gamma$, is described by the asymptotic formula

$$
Q^{(0)}(\Omega)=Q^{(\mathrm{ZDP})}(\Omega)=C_{\text {scale }} S\left(\frac{\Omega-\omega_{m}}{\Delta \omega}\right),
$$

where

$$
\begin{gathered}
S(x)=|\operatorname{Re}[\widetilde{S}(x)]| \\
\widetilde{S}(x)=\int_{0}^{\infty} d \tau \frac{\exp (-i x \tau)}{\{(1-i) \sinh [(1-i) \tau]\}^{1 / 2}},
\end{gathered}
$$

and $C_{\text {scale }}$ and $\Delta \omega$ are frequency-independent scale factors,

$$
\begin{gathered}
C_{\text {scale }}=\frac{4 \sqrt{\pi}\left|q_{1 m}\right|^{2}}{\omega_{m}\left(\left|\omega^{\prime \prime}\right|\right)^{3 / 4}} \frac{\exp \left(-E_{m} / T\right)}{Z\left(\Gamma T \overline{q_{m}^{2}}\right)^{1 / 4}}, \\
\Delta \omega=\operatorname{sgn}\left(\omega^{\prime \prime}\right)\left(\Gamma\left|\omega^{\prime \prime}\right| T \overline{\dot{q}_{m}^{2}}\right)^{1 / 2}, \\
\omega^{\prime \prime} \equiv \frac{d^{2} \omega\left(E_{m}\right)}{d E_{m}^{2}},
\end{gathered}
$$

where $\omega(E)$ is the frequency of an eigenoscillation as a function of its energy,

$$
E=\frac{1}{2} \dot{q}^{2}+U(q)
$$

and $E_{m}$ is the energy at which $\omega(E)$ has an extremum,

$$
Z=2 \pi \int_{U_{\min }}^{\infty} d E \frac{\exp (-E / T)}{\omega(E)}
$$

is the partition function, $U_{\min }$ is the minimum value of $U(q)$, $\overline{\dot{q}_{m}^{2}}$ is $\dot{q}^{2}$ averaged over one period of the motion when $E=E_{m}$, and $q_{1 m} \equiv q_{1}\left(E_{m}\right)$ is the first Fourier component in the expansion of $q$ as a periodic function of angle $\phi$ [19] at the energy $E_{m}$,

$$
q=\sum_{n=-\infty}^{\infty} q_{n}(E) \exp (-i n \phi) .
$$

As shown in Fig. 1, the function $S(x)$ has an asymmetric peak whose height, half-width, and position have characteristic values of order unity.

Equations (6)-(10) are valid provided that (i) the ZDP is well separated from other characteristic peaks in the spectrum; (ii) there is a homogeneous population of the relevant energy band around $E_{m}$; (iii) the ZDP is larger than the relaxational plateau of the spectrum. Thus the conditions for the validity of Eqs. (6)-(10) are (cf., [13])

$$
\begin{aligned}
& \left|\frac{\Delta \omega}{\min \left(\omega_{m},\left|\omega_{m}-\omega_{i}\right|\right)}\right|,\left|\frac{\Delta \omega}{\omega^{\prime \prime} T^{2}}\right|^{1 / 2} \ll 1, \\
& -|\Delta \omega| \lesssim \operatorname{sgn}\left(\omega^{\prime \prime}\right)\left(\Omega-\omega_{m}\right) \ll\left|\omega^{\prime \prime}\right| T^{2},
\end{aligned}
$$




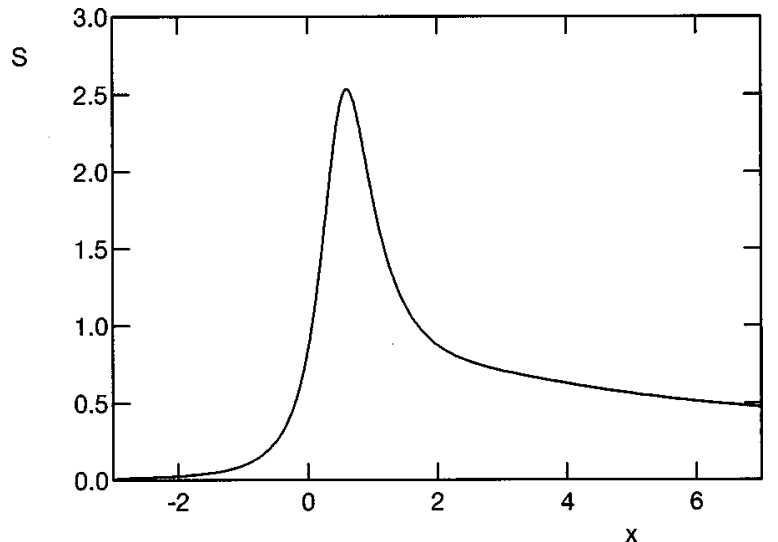

FIG. 1. The universal shape of zero-dispersion peaks, as given by the function $S(x)$ defined in Eq. (6).

$$
T>\frac{0.4 E_{m}}{\ln \left|\omega^{\prime \prime} E_{m}^{2} / \Delta \omega\right|},
$$

where $\omega_{i}$ denotes the frequency of any other characteristic peak of the spectrum. These conditions are always satisfied in the asymptotic limit of small $\Gamma$ when $\Omega$ is close enough to $\omega_{m}$.

It may be noted from Eqs. (6)-(10), first, that the height and width of the ZDP become infinitely large and small, respectively, as $\Gamma \rightarrow 0$ and, secondly, that the dependence of the height on $T$ is of activation type, i.e., the height grows extremely fast with $T$ if $T \ll E_{m}$. Thus one may expect SR to be manifested in the system. Indeed, if Eqs. (6)-(10) are inserted into Eq. (4) with account taken of Eq. (11),

$$
\begin{gathered}
\mathcal{R}=\mathcal{R}^{(\mathrm{ZD})} \equiv \frac{1}{4} A^{2} \frac{\pi^{2} \omega_{m}^{2}}{T^{2}} C_{\text {scale }} R\left(\frac{\Omega-\omega_{m}}{\Delta \omega}\right), \\
R(x)=\frac{|\widetilde{S}(x)|^{2}}{S(x)} .
\end{gathered}
$$

The function $R(x)$, plotted in Fig. 2 characterizes the frequency dependence of the SNR. It decreases monotonically with $x$ (cf. the harmonic oscillator for which $\mathcal{R} \propto \Omega^{-2}$ ), but

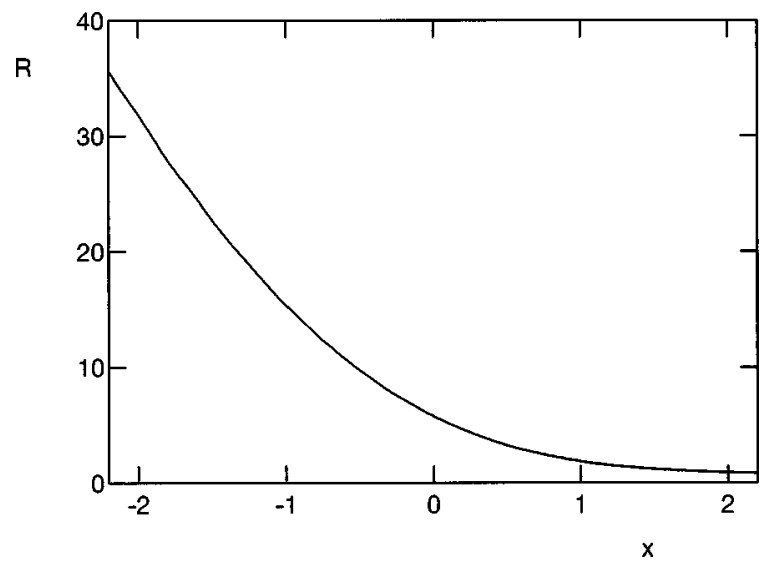

FIG. 2. The normalized frequency dependence of the signal-tonoise ratio in the asymptotic limit of small dissipation, as given by the function $R(x)$ defined in Eq. (12). the form of the decrease changes with $x$ : it can be shown that $\mathcal{R} \propto(-x)^{3 / 2}$ for large negative $x$, and that SNR $\propto x^{-1 / 2}$ for large positive $x$.

Of greater interest in the present context is that the SNR is proportional to $C_{\text {scale }}$, which increases sharply with $T$ for $T \ll E_{m}$. Consequently, the SNR must increase with $T$ within some range [20], just as in the case of SR in conventional bistable systems $[5-7,1]$. The same holds true even when the shape of the ZDP is not universal. The only condition is that the magnitude of the ZDP should be sufficiently large in comparison to other spectral peaks [8]. The activation-law type dependence of the SNR on $T$ arises because both the susceptibility and fluctuation spectrum are determined by those oscillator vibrations whose energies lie within a narrow band around the extremal frequency, whose population increases rapidly with $T$.

The frequency dependence of the SNR is well described by the universal function $R$, but only for very small values of $\Gamma$ and in the close vicinity of $\omega_{m}$ (see Sec. V); the same restrictions apply also to the shape of the ZDP itself $[13,15]$. In order to calculate the SNR over a wider range of parameters, it is necessary to compute the fluctuation spectrum numerically. The algorithm we have used for this calculation is described in the next section.

\section{THEORY OF FLUCTUATION SPECTRA FOR UNDERDAMPED MOTION}

An efficient algorithm for the calculation of fluctuation spectra in underdamped oscillators was developed in [21], but was restricted to systems with single-well potentials. Because the potential that describes the dynamics of SQUIDs can be of either the single-well or multiwell type, it is necessary to generalize the method of [21] to treat the case where the potential may also have one or more local barriers.

Our aim, therefore, is to calculate $Q^{(0)}(\Omega)$, i.e., the fluctuation spectrum (5) of the coordinate $q$ whose dynamics is governed by Eq. (1) with $A=0$. It is convenient to start from the Fokker-Planck equation (FPE) for the probability density $w\left(q, p, t ; q_{0}, p_{0}, 0\right)$ of a transition in the space of coordinates $q$ and momenta $p \equiv \dot{q}$ from the point $\left(q_{0}, p_{0}\right)$ occupied at time zero to the point $(q, p)$ occupied at time $t$ (see, e.g., [22]),

$$
\begin{gathered}
\frac{\partial w}{\partial t}=-\frac{\partial}{\partial q}(p w)+\frac{\partial}{\partial p}\left(\frac{d U(q)}{d q} w\right)+\Gamma \hat{L} w, \\
\hat{L}=+\frac{\partial}{\partial p} p+T \frac{\partial^{2}}{\partial p^{2}}, \quad w \equiv w\left(q, p, t ; q_{0}, p_{0}, 0\right), \\
w\left(q, p, 0 ; q_{0}, p_{0}, 0\right)=\delta\left(q-q_{0}\right) \delta\left(p-p_{0}\right) .
\end{gathered}
$$

According to the definition (5), we can express the time correlation function $\widetilde{Q}(t)$ in terms of $w\left(q, p, t ; q_{0}, p_{0}, 0\right)$ and the stationary distribution $w_{\text {st }}\left(q_{0}, p_{0}\right)$ as

$$
\widetilde{Q}(t)=\int_{-\infty}^{\infty} \int_{-\infty}^{\infty} d q d p(q-\langle q\rangle) \widetilde{W}(q, p, t),
$$



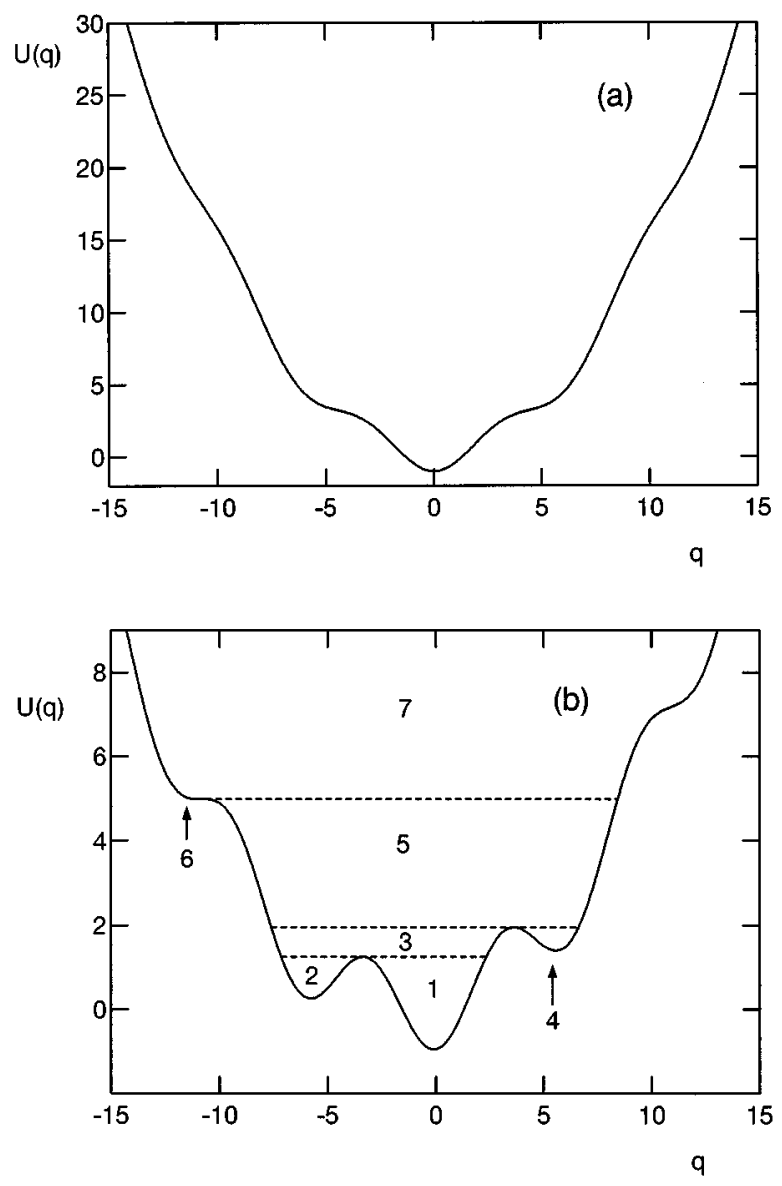

FIG. 3. The effective potential for a SQUID, Eq. (26), plotted for (a) $B=0.3, q_{\mathrm{dc}}=0$; (b) $B=0.1, q_{\mathrm{dc}}=-1.0$. The numbers in (b) identify different regions of phase space corresponding to motion confined within energy ranges of three different kinds, separated by the dashed lines: between local energy maxima adjacent in height; between a local maximum and the local minimum adjacent in coordinate; or between the highest local maximum and infinity.

$$
\begin{array}{r}
\widetilde{W}(q, p, t)=\int_{-\infty}^{\infty} \int_{-\infty}^{\infty} d q_{0} d p_{0}\left(q_{0}-\langle q\rangle\right) \\
\times w\left(q, p, t ; q_{0}, p_{0}, 0\right) w_{\mathrm{st}}\left(q_{0}, p_{0}\right), \\
w_{\mathrm{st}}(q, p)=Z^{-1} \exp (-E / T) .
\end{array}
$$

Like $w\left(q, p, t ; q_{0}, p_{0}, 0\right)$, the function $\widetilde{W}(q, p, t)$ satisfies the FPE (13), but with a different initial condition

$$
\widetilde{W}(q, p, 0)=(q-\langle q\rangle) w_{\text {st }}(q, p) .
$$

For further analysis, it is convenient to rewrite Eq. (13) in an energy-angle representation [19] and to make a half Fourier transform with respect to time. Unlike the single-well case [21], however, the energy-angle representation must be differently defined in different regions of the phase space, divided by separatrices corresponding to the barrier energy levels [cf. Fig. 3(b)].

Tagging each such region with an index $j$, we can write the equation for the half Fourier transform $W$ in the $j$ th phase space region as

$$
\begin{gathered}
-i \Omega W+\omega \frac{\partial W}{\partial \phi}=\Gamma \hat{L} W+(q-\langle q\rangle) w_{\mathrm{st}}(q, p), \\
\omega \equiv \omega^{(j)}(E), \quad q \equiv q^{(j)}(E, \phi), \quad p \equiv p^{(j)}(E, \phi), \\
\hat{L} \equiv \hat{L}^{(j)}(E, \phi)=\left[p \frac{\partial}{\partial E}-\omega \frac{\partial q}{\partial E} \frac{\partial}{\partial \phi}\right. \\
\times\left[p\left(1+T \frac{\partial}{\partial E}\right)-T \omega \frac{\partial q}{\partial E} \frac{\partial}{\partial \phi}\right], \\
W \equiv W^{(j)}(E, \phi, \Omega)=\int_{0}^{\infty} d t \exp (i \Omega t) \widetilde{W}(q, p, t) .
\end{gathered}
$$

Like $q(E, \phi)$ and $p(E, \phi)$, the function $W(E, \phi ; \Omega)$ is periodic in $\phi$ with period $2 \pi$, and so can be expanded in a Fourier series,

$$
W^{(j)}(E, \phi ; \Omega)=\sum_{n=-\infty}^{\infty} W_{n}^{(j)}(E, \Omega) \exp (\text { in } \phi) .
$$

Substituting Eq. (17) into Eq. (16), we obtain

$$
\begin{gathered}
-\left(\Omega-n \omega^{(j)}\right) W_{n}^{(j)}=\Gamma \sum_{m} \hat{L}_{n m}^{(j)} W_{m}^{(j)}+\left(q_{n}^{(j)}-\langle q\rangle \delta_{n 0}\right) w_{\mathrm{st}}, \\
\hat{L}_{n m}^{(j)}=\frac{1}{2 \pi} \int_{0}^{2 \pi} d \phi \exp (-i n \phi) \hat{L}^{(j)} \exp (i m \phi), \\
q_{n}^{(j)}=\frac{1}{2 \pi} \int_{0}^{2 \pi} d \phi \exp (-i n \phi) q^{(j)}(E, \phi),
\end{gathered}
$$

where $\delta_{n 0}$ is a Kronecker delta. Our aim is to find the $W_{n}^{(j)}$, since the fluctuation spectrum can be written in terms of them as

$$
\begin{aligned}
Q^{(0)}(\Omega)= & \sum_{j} 2 \operatorname{Re}\left[\int_{E_{\min }^{(j)}}^{E^{(j)}} \frac{d E}{\omega^{(j)}(E)}\right. \\
& \left.\times \sum_{n=0}^{\infty}\left\{\left[q_{n}^{(j)}(E)\right]^{*}-\delta_{n 0}\langle q\rangle\right\} W_{n}^{(j)}(E, \Omega)\right],
\end{aligned}
$$

where $E_{\min }^{(j)}$ and $E_{\max }^{(j)}$ are, respectively, the minimum and maximum energies of the $j$ th region.

If we consider the underdamped case ( is usually the case, it is the peaks of $Q(\Omega)$ that are of interest rather than low fluctuational plateaus, then we need retain only the diagonal terms in Eq. (18) (i.e., terms $\propto \hat{L}_{n n}$ ) [21], thus obtaining a closed set of ordinary differential equations for $W_{n}^{(j)}[23]$, 


$$
\begin{aligned}
-i(\Omega-n \omega) W_{n}^{(j)}= & \Gamma\left(1+\overline{p^{2}} \frac{d}{d E}\right)\left(1+T \frac{d}{d E}\right) W_{n}^{(j)} \\
& -\Gamma \operatorname{Tn}^{2} \omega^{2} \overline{\left(\frac{\partial q}{\partial E}\right)^{2}} W_{n}^{(j)} \\
& +\left(q_{n}-\delta_{n 0}\langle q\rangle\right) w_{\mathrm{st}}
\end{aligned}
$$

where the bar implies averaging $\left[(1 / 2 \pi) \int_{0}^{2 \pi} d \phi \cdots\right]$ over the angle.

In order to solve Eq. (20), we also need to know the boundary conditions at $E_{\min }^{(j)}$ and $E_{\max }^{(j)}$ for each region. If $n$ $\neq 0[25], W$ vanishes at the boundaries

$$
W_{n}^{(j)}\left(E_{\min }^{(j)}, \Omega\right)=W_{n}^{(j)}\left(E_{\max }^{(j)}, \Omega\right)=0 .
$$

The case where $E_{\min }^{(j)}$ corresponds to a local minimum in the potential may be treated similarly to the single-well case [21] in deriving Eq. (21): Eq. (20) can have two solutions near the minimum, $W_{n}^{(j)} \propto\left(E-E_{\mathrm{min}}^{(j)}\right)^{1 / 2}$ or $W_{n}^{(j)} \propto\left(E-E_{\mathrm{min}}^{(j)}\right)^{-1 / 2}$, given the necessary finiteness of $W_{n}^{(j)}$, we choose the convergent solution and thus arrive at Eq. (21). The validity of the condition is obvious when $E_{\max }^{(j)}$ is equal to infinity. When $E_{\min }^{(j)}$ or $E_{\max }^{(j)}$ correspond to a barrier, Eq. (21) is derived in the following way. It is straightforward to demonstrate that, for energies close to one of the barrier levels $E_{b}$,

$$
\overline{\left(\frac{\partial q}{\partial E}\right)^{2}} \propto \frac{1}{\left(\left|E-E_{b}\right|\right)^{2}} .
$$

The solution of Eq. (20) near the barrier can thus be of two types: $W_{\text {convergent }} \propto\left|E-E_{b}\right|$ and $W_{\text {divergent }} \propto\left(\left|E-E_{b}\right|\right)^{-1}$. Allowing for the finiteness of $W_{n}^{(j)}$ as before, we choose the convergent solution and arrive at Eq. (21).

Equation (20) can easily be solved numerically (cf. [21]) for the boundary conditions (21), enabling the power spectrum, susceptibility, and SNR to be calculated via Eqs. (19) and (4), respectively; the algorithm for calculation of certain parameters in Eqs. (19), (20) follows directly from their definition and is described in detail in $[15,21]$. In Secs. IV and V below we report the results of such calculations for a system of practical importance, the SQUID, and compare them with measurements made with an analog electronic model.

\section{ANALOG ELECTRONIC EXPERIMENTS FOR A SQUID MODEL}

The aim was to try to test some of the ideas of the preceding section by modeling a superconductive loop containing a Josephson junction. This is the simplest type of SQUID. It forms the central element of a radio-frequency SQUID, is often used in more complicated SQUID devices [17], and, as we shall see, may be expected to display ZDSR for an appropriate choice of parameters.

The dynamics of the loop is described in many cases by the resistively shunted model, in terms of which the time evolution of the phase of the order parameter, or of the magnetic flux $\Phi(t)$ threading the loop, can [17] be described by the equation

$$
\begin{gathered}
L C \frac{d^{2} q}{d \tau^{2}}+\frac{L}{R_{N}} \frac{d q}{d \tau}+q+\beta \sin (q)=q_{e}, \\
q=2 \pi \frac{\Phi}{\Phi_{0}}, \quad q_{e}=2 \pi \frac{\Phi_{e}}{\Phi_{0}}, \quad \beta=\frac{2 \pi L J_{c}}{\Phi_{0}} .
\end{gathered}
$$

Here $\Phi$ is the full magnetic flux through the loop; $\Phi_{e}$ is the flux of the external magnetic field; $\Phi_{0}=h / 2 e$ is the flux quantum; $L$ is the inductance of the loop; and $C, R_{N}$, and $J_{c}$ are, respectively, the capacitance, normal resistivity, and critical supercurrent of the junction.

The external flux usually includes a noisy component $\Phi_{N}(\tau)$, to which can formally be added an additional contribution due to thermal fluctuations within the loop itself and to noise in the Josephson junction. In addition, there is often a constant component $\Phi_{d c}$ and a small periodic signal $\Phi_{s} \cos \left(\omega_{s} \tau\right)$. We will therefore assume that

$$
\begin{gathered}
\Phi_{e} \equiv \Phi_{e}(\tau)=\Phi_{d c}+\Phi_{s} \cos \left(\omega_{s} \tau\right)+\Phi_{N}(\tau) \\
\left\langle\Phi_{N}(\tau) \Phi_{N}\left(\tau^{\prime}\right)\right\rangle=2 D \delta\left(\tau-\tau^{\prime}\right)
\end{gathered}
$$

Taking account of Eq. (24) and introducing the normalized variables

$$
\begin{gathered}
t=\omega_{p} \tau, \quad \Gamma=\frac{1}{\omega_{p} R_{N} C}, \quad \Omega=\frac{\omega_{s}}{\omega_{p}} \\
A=\frac{\Phi_{s}}{L J_{c}}, \quad q_{\mathrm{dc}}=\frac{2 \pi \Phi_{\mathrm{dc}}}{\Phi_{0}} \\
T=\frac{2 \pi D R_{N}}{\Phi_{0} L^{2} J_{c}}, \quad \omega_{p}=\left(\frac{2 \pi J_{c}}{C \Phi_{0}}\right)^{1 / 2}
\end{gathered}
$$

Eq. (23) takes the same form as Eq. (1), with

$$
U(q)=\frac{B}{2}\left(q-q_{\mathrm{dc}}\right)^{2}-\cos (q), \quad B \equiv \frac{1}{\beta} .
$$

The frequency of eigenoscillations in the potential $U(q)$ of Eq. (26) possesses extrema as a function of energy if $B \leqq 1$ (Fig. 4). Consequently, the system should display the full range of zero-dispersion phenomena including, in particular, ZDSR. The criterion for the smallness of the amplitude of the periodic signal can in practice easily be found experimentally by finding the maximum amplitude for which the response is linear.

The circuit used to model Eqs. (1) and (26), shown in outline by the block diagram of Fig. 5, was designed according to a standard prescription [27,28], using operational amplifiers, an analog multiplier [29], and a trigonometric integrated circuit (IC) [30] to perform the necessary mathematical operations. In the diagram $A^{\prime} \cos \Omega^{\prime} t^{\prime}$ and $f^{\prime}\left(t^{\prime}\right)$ are, respectively, a signal and an external noise applied to the underdamped nonlinear oscillator. $A^{\prime}$ is the amplitude of the signal in volts, $f^{\prime}\left(t^{\prime}\right)$ is the value of the noise voltage applied to the circuit, and $\Omega^{\prime}$ and $t^{\prime}$ are the real frequency and time. Setting to zero the total currents at the inputs of the operational amplifiers whose outputs are $V_{A}$ and $V_{B}$, respectively, we obtain 

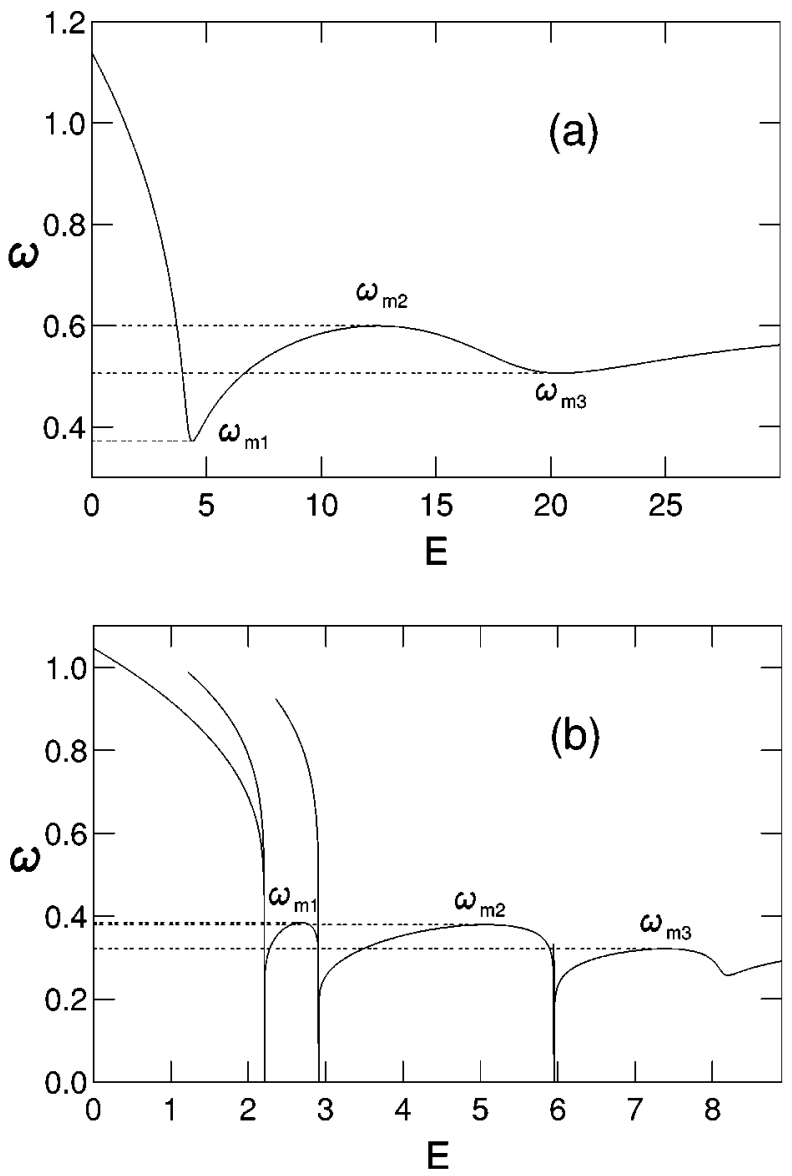

FIG. 4. Dependences of the frequency $\omega(E)$ of eigenoscillation on energy $E$ for the potentials shown in Fig. 3: (a) $B=0.3, q_{\mathrm{dc}}=0$; (b) $B=0.1, q_{\mathrm{dc}}=-1.0$. Dashed lines indicate the positions of the first three extrema in each case: (a) $\omega_{m 1}=0.372, \omega_{m 2}=0.600$, $\omega_{m 3}=0.506$; (b) $\omega_{m 1}=0.385, \omega_{m 2}=0.380, \omega_{m 3}=0.321$.

$$
\begin{gathered}
\frac{V_{A}}{R_{G}}+C_{1} \frac{d V_{A}}{d t^{\prime}}+\frac{f^{\prime}\left(t^{\prime}\right)}{R_{N}}+\frac{A^{\prime} \cos \left(\Omega^{\prime} t^{\prime}\right)}{R_{F}} \\
-\frac{\left(R_{5} / R_{4}\right) V_{B}}{R_{2}}+\frac{V_{D}}{R_{1}}=0, \\
C_{2} \frac{d V_{B}}{d t^{\prime}}+\frac{V_{A}}{R_{3}}=0 .
\end{gathered}
$$

The trigonometric IC was configured to give an output of $10 \sin \left[50\left(y_{1}-y_{2}\right)\right]$ where the two inputs $y_{1}$ and $y_{2}$ are in volts and the argument of the sine is in degrees. The IC operation is restricted to lie within the range $\pm 500^{\circ}$. In order to increase the dynamic range of $q$ [see Eq. (26)] encompassed by the model, an analog multiplier was used as shown to convert the argument to the double angle. The voltage at its output, in terms of the voltage $V_{C}$ at the input of the trigonometric IC and the constant voltages $V_{1}$ and $V_{2}$, and allowing for internal scaling by a factor of 0.1 , is

$$
V_{D}=0.1\left\{10 \sin \left[50\left(V_{1}-V_{C}\right)\right]\right\}^{2}+V_{2}
$$

or, in terms of the double angle, now expressed in radians,

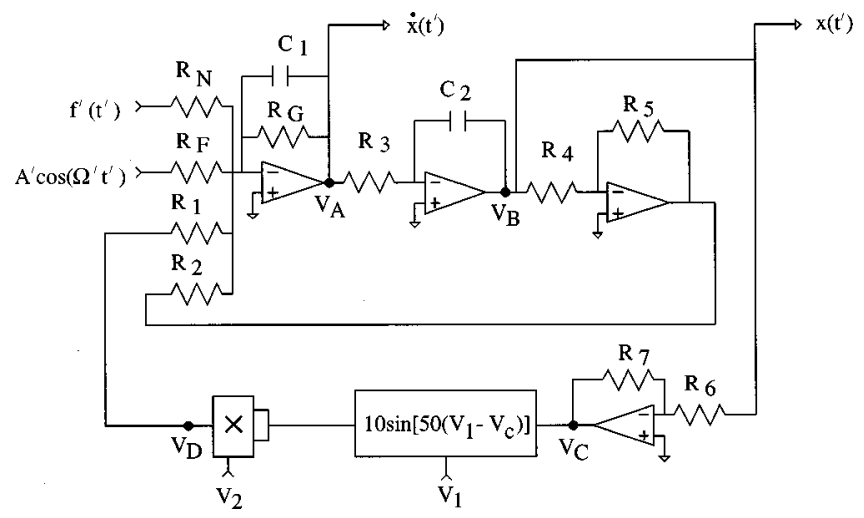

FIG. 5. Block diagram of the analog electronic circuit model. Its behavior can conveniently be analyzed in terms of the voltages $V_{A}, V_{B}, V_{C}$, and $V_{D}$ at the points indicated (see text).

$$
V_{D}=5\left(1-\cos \left[\frac{\pi}{1.8}\left(V_{1}-V_{C}\right)\right]\right)+V_{2} .
$$

The voltage $V_{C}$ is just

$$
V_{C}=-\frac{R_{7}}{R_{6}} V_{B}
$$

Eliminating $V_{A}, V_{C}$, and $V_{D}$ from Eqs. (27), (28), (30), and (31), and writing $V_{B} \equiv x$, the differential equation for the voltage $x$ in the circuit can therefore be written

$$
\begin{gathered}
R_{1} C_{1} R_{3} C_{2} \frac{d^{2} x}{d t^{\prime 2}}+\frac{R_{1}}{R_{G}} R_{3} C_{2} \frac{d x}{d t^{\prime}}+\frac{R_{1} R_{5}}{R_{2} R_{4}} x \\
-5\left\{1-\cos \left[\frac{\pi}{1.8}\left(V_{1}+\frac{R_{7}}{R_{6}} x\right)\right]\right\}-V_{2} \\
=\frac{R_{1}}{R_{F}} A^{\prime} \cos \Omega^{\prime} t^{\prime}+\frac{R_{1}}{R_{N}} f^{\prime}\left(t^{\prime}\right),
\end{gathered}
$$

where we have chosen

$$
\begin{gathered}
R_{N}=R_{F}=100 \mathrm{k} \Omega, \quad R_{1}=R_{3}=100 \mathrm{k} \Omega, \\
R_{4}=R_{5}=R_{6}=10 \mathrm{k} \Omega, \quad R_{7}=11.459 \mathrm{k} \Omega, \\
R_{G}=22 \mathrm{M} \Omega, \quad C_{1}=C_{2}=10 \mathrm{nF}, \quad V_{1}=-0.9 \mathrm{~V} .
\end{gathered}
$$

The multiwell and single-well cases of the potential (26) correspond to different values of the parameters $R_{2}$ and $V_{2}$. For example, on introducing $R_{2}=100 \mathrm{k} \Omega, V_{2}=-3.93 V$, the time constant $\tau^{\prime}=R_{1} C_{1} / \sqrt{5}=R_{3} C_{2} / \sqrt{5}$, and the damping constant $\Gamma^{\prime}=R_{1} /\left(R_{G} \sqrt{5}\right)$, Eq. (32) can be reduced to

$$
\begin{aligned}
\tau^{\prime 2} \ddot{x}+ & \Gamma^{\prime} \tau^{\prime} \dot{x}+0.2(x-1.07)+\sin (2 x) \\
& =0.2 A^{\prime} \cos \Omega^{\prime} t^{\prime}+0.2 f^{\prime}\left(t^{\prime}\right),
\end{aligned}
$$

whose parameters are readily related to those in the model (1) by means of the scaling relations 


$$
\begin{gathered}
q=2 x, \quad \tau=\frac{\tau^{\prime}}{\sqrt{2}}, \quad t=\frac{t^{\prime}}{\tau}, \quad \Omega=\Omega^{\prime} \tau, \quad \Gamma=\frac{\Gamma^{\prime}}{\sqrt{2}}, \\
B=0.1, \quad q_{e}=2.14 \\
A=0.2 A^{\prime}, \quad f(t)=0.2 f^{\prime}\left(t^{\prime}\right) .
\end{gathered}
$$

The nominal value of $\Gamma$ was 0.00144 . However, for such small damping, the actual value usually differs from the nominal one due to the effects of stray capacitance and other nonidealities of the circuit. In the present case, the actual value, measured experimentally by two independent methods [15], was found to be $\Gamma=0.0012$ for the multiwell case and $\Gamma=0.0011$ for the single-well case.

The circuit model was driven by noise from a feedback shift-register noise generator [27,31] and by a sinusoidal periodic force from a Hewlett-Packard model 3325B frequency synthesizer. The response of the circuit to stochastic and periodic forces was analyzed with the aid of a Microstar DAP3200a/415 ADC card [32] installed in a Pentium 133 personal computer.

The evolution of the fluctuation spectra with increasing noise intensity was measured for the two cases of a singlewell and a multiwell potential (with four wells) as shown in Figs. 3(a) and 3(b). The results are shown in Fig. 6.

The SNR was calculated as a function of both noise intensity and signal frequency, for both single-well and multiwell cases, yielding the results shown in Fig. 7. The dependences of the SNR on $T$ for fixed $\Omega$, and on $\Omega$ for fixed $T$, both measured and calculated, are shown in Figs. 8 and 9, respectively.

\section{DISCUSSION}

It is immediately evident from the results of Figs. 7 and 8 that a substantial noise-induced increase in the SNR can occur for the SQUID model: note the satisfactory agreement between the theory and the analog experimental data (Fig. 8). It is to be expected, therefore, that underdamped SQUIDs will display closely similar behavior.

The special features of ZDSR peculiar to SQUIDs are closely related to corresponding features in their fluctuation spectra which, in turn, are determined by the characteristic form of $\omega(E)$. The latter may have several extrema, as shown in Fig. 4. Thus there could be several ZDPs corresponding to the different extrema. They become activated sequentially with increasing $T$. This is most clearly seen in the single-well case where the extremal frequencies are well separated. The higher the extremal energy, the higher the noise intensity at which the spectral contribution from a ZDP becomes comparable with those from other energy ranges. At the same time, the higher the extremal energy $E$, the larger the amplitude of the oscillations and, in most cases, the flatter $\omega(E)$ becomes. The latter effect broadens the range of energies that contribute significantly to the ZDP $[12,13,15]$ and causes the correlation time of the oscillation to lengthen. Taken together, the two effects result in the maximum magnitude of each successive ZDP increasing markedly [cf. Fig. 6(a)].

In the case of a multiwell potential $(B \ll 1)$, most of the extremal eigenfrequencies are very close to each other (all $\approx B^{1 / 2}$ ). Thus, in addition to the increase in magnitude of each successive partial ZDP, many of the ZDPs will overlap; and the higher the noise intensity, the greater the number of overlapping peaks. Thus the noise-induced increase in the magnitude of the spectral peak is especially pronounced in the multiwell case. As shown by the analysis of $C_{\text {scale }}(7)$ for small $B$, the maximum magnitude of the peak grows as $B$ decreases, as $\propto B^{-3 / 2}$, until it reaches the limit $\sim \Gamma^{-1}$.

Let us now turn to the signal-to-noise ratio. As discussed in Sec. II, if a ZDP in the spectrum $Q^{(0)}(\Omega)$ dominates over other contributions to the spectrum in the vicinity of $\omega_{m}$ then the SNR is proportional to $C_{\text {scale }} / T^{2}$ in the vicinity of $\omega_{m}$, which provides a resonance-like dependence of the SNR on $T$ : it first sharply increases with $T$, like the ZDP magnitude, and then, when the temperature becomes of the order of $E_{m}$, the sharp growth of $C_{\text {scale }}$ with $T$ saturates and the increase of the SNR changes into a slow decrease. Although the spectrum in a SQUID typically manifests more than one ZDP, and the ZDPs often overlap (cf. Fig. 6), the dependence of the SNR on $T$ may still be characterized qualitatively (though not quantitatively) by Eq. (12) using parameters of that extremum of $\omega(E)$ corresponding to the ZDP which dominates within the relevant ranges of temperature and frequency. Figure 8 demonstrates resonancelike dependences of the SNR on $T$. The rapid increase of the SNR as $T$ decreases at small $T$ occurs because the SNR is determined at small temperatures by oscillations near the bottom of the lowest potential well, resulting in a dependence proportional to $T^{-1}$. Thus, when the signal frequency is close to one of the extreme eigenfrequencies, the temperature dependence of the SNR is very similar to that for conventional SR $[5,6,1,2]$.

It should be emphasized that an increase of SNR with $T$ would still have been observed, even if the damping constant had been considerably larger than the value $\left(\Gamma \sim 10^{-3}\right)$ used in the present experiments: the relevant criterion for $\Gamma$ can be written approximately as

$$
\Gamma \ll B^{1 / 2} \lesssim 1
$$

or, in terms of SQUID parameters,

$$
R_{N} \gg(L / C)^{1 / 2}, \quad \beta \gtrsim 1 .
$$

A characteristic feature of the frequency dependence of the SNR in a SQUID is its rapid increase in the direction of a ZDP's steepest decrease. The reason is that the real part of the susceptibility (and also $|\chi|^{2}$ ) decreases much more slowly than the spectral density [c.f. Eq. (12)]. As a rule, the SNR passes through a maximum and then decreases again. The physical reason is that the spectral density is then being determined by energy regions far from the extremal energy: the SNR increase therefore saturates and changes to a decrease as the signal frequency moves even further away from the extremal eigenfrequency. Note that the frequency range within which the SNR increases is typically much larger than the width of the associated ZDP itself.

The frequency dependence of the SNR is described quite well by our numerical algorithm, as can be seen in Fig. 9. In Fig. 9(a), which corresponds to the monostable SQUID, the left (sharply decreasing) section of the dependence is mostly due to the minimum of $\omega(E)$ at about 0.4 ; the left wing of the peak is mostly due to the ZDP at $\Omega \approx 0.6$ while the right 
(a)

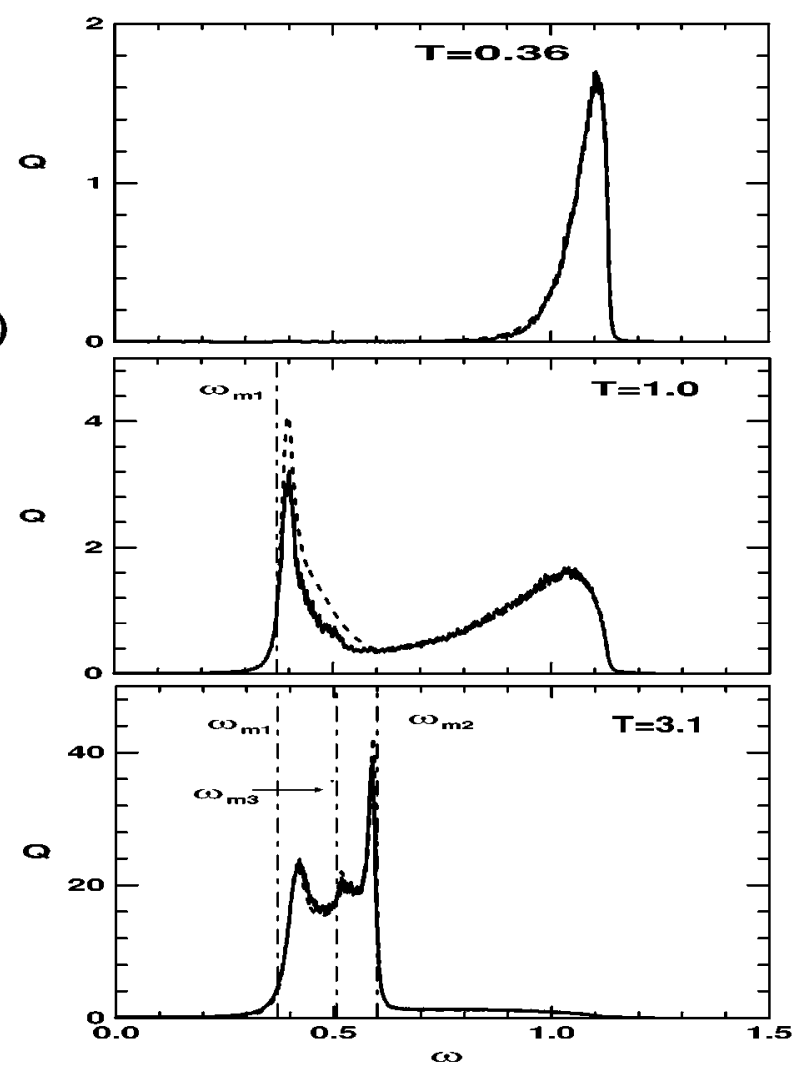

(b)

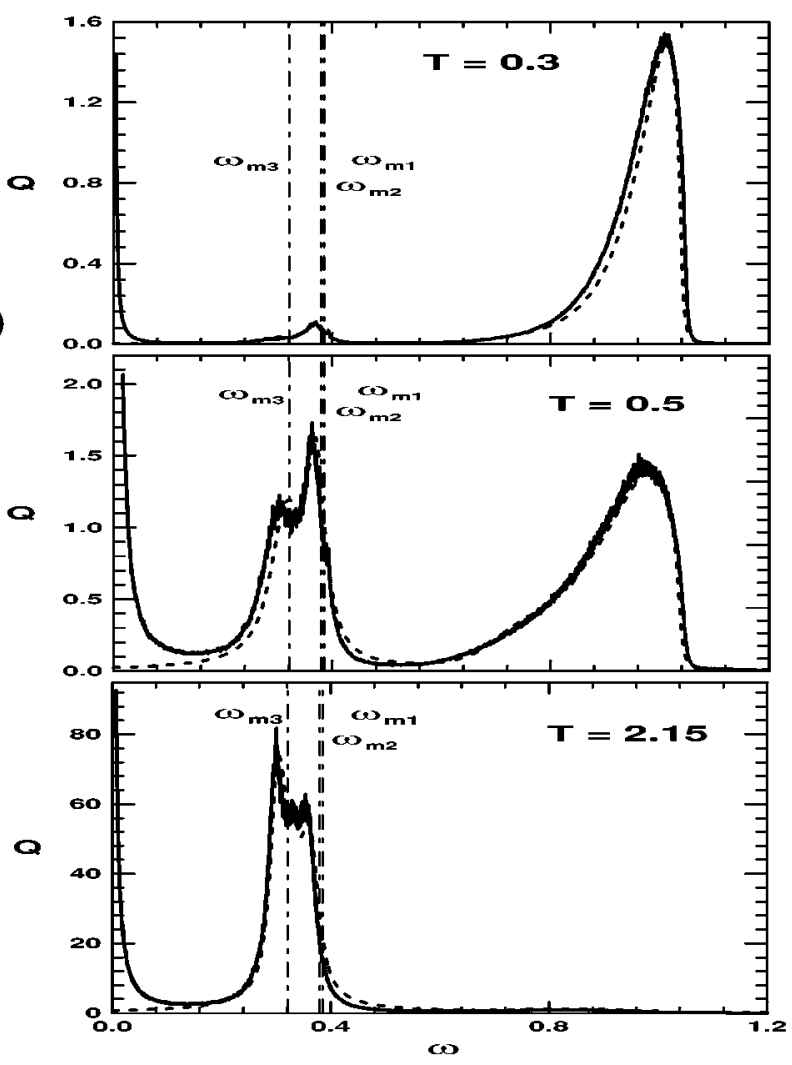

FIG. 6. Evolution of the fluctuation spectra with increase of noise intensity $T$, measured (full curves) and calculated using the numerical algorithm of Sec. III (dashed curves) for (a) the singlewell SQUID potential of Fig. 3(a); and (b) the multiwell SQUID potential of Fig. 3(b). Dash-dotted lines mark the positions of the relevant extrema of $\omega(E)$ (see Fig. 4). (a)

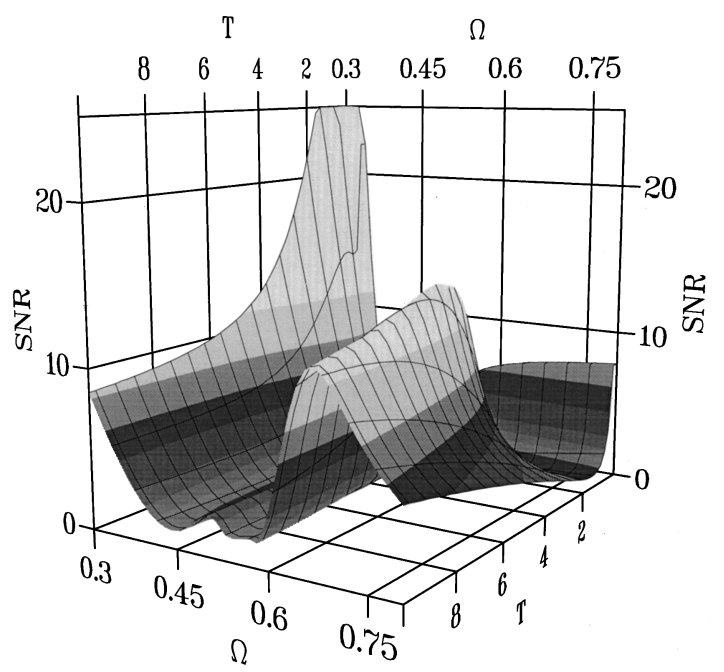

(b)

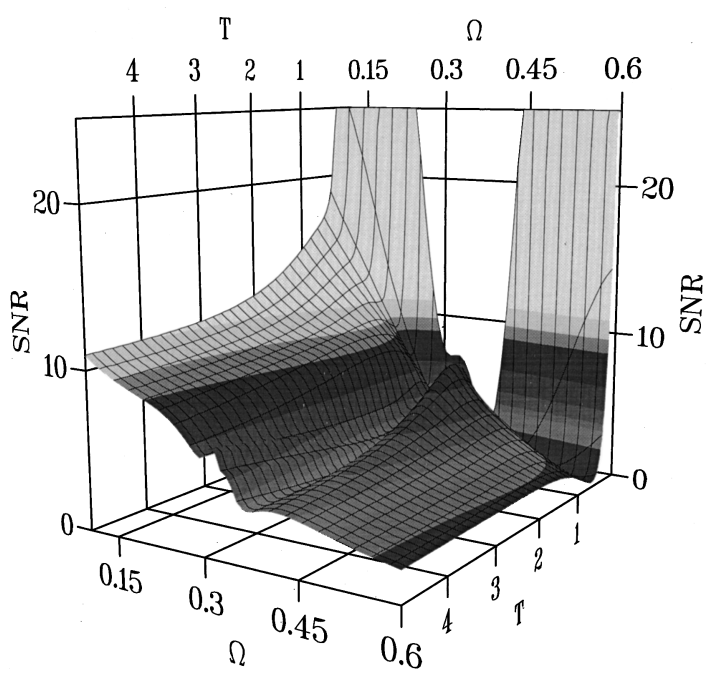

FIG. 7. The dependence of the signal-to-noise ratio (normalized by a squared signal amplitude) on the noise intensity $T$ and the signal frequency $\Omega$, calculated using Eq. (4) and the numerical algorithm described in Sec. III, for (a) the single-well SQUID potential of Fig. 3(a); and (b) the multiwell SQUID potential of Fig. $3(b)$.

(decreasing) wing of the peak is due to the relaxational plateau dominating in the spectrum at these frequencies. Figure 9(b), which corresponds to the multistable SQUID, exhibits two peaks in the SNR. The left-hand wing of the right-hand peak is associated with the ZDP near $\Omega=0.38$, whereas the opposite wing of this peak is associated with the intrawell peak of the spectrum. The decrease of the SNR as the frequency decreases changes at $\Omega \approx 0.3$ into an increase, contrary to Eq. (12), because the spectrum in this range of frequencies is governed by energies close to one of the barrier levels where the parabolic approximation is inapplicable and the spectrum decreases much more rapidly than predicted by Eq. (6). The opposite wing of this peak of the SNR dependence is associated with the zero-frequency peak of the spectrum [33].

Thus, the frequency dependence of the SNR in ZD oscil- 

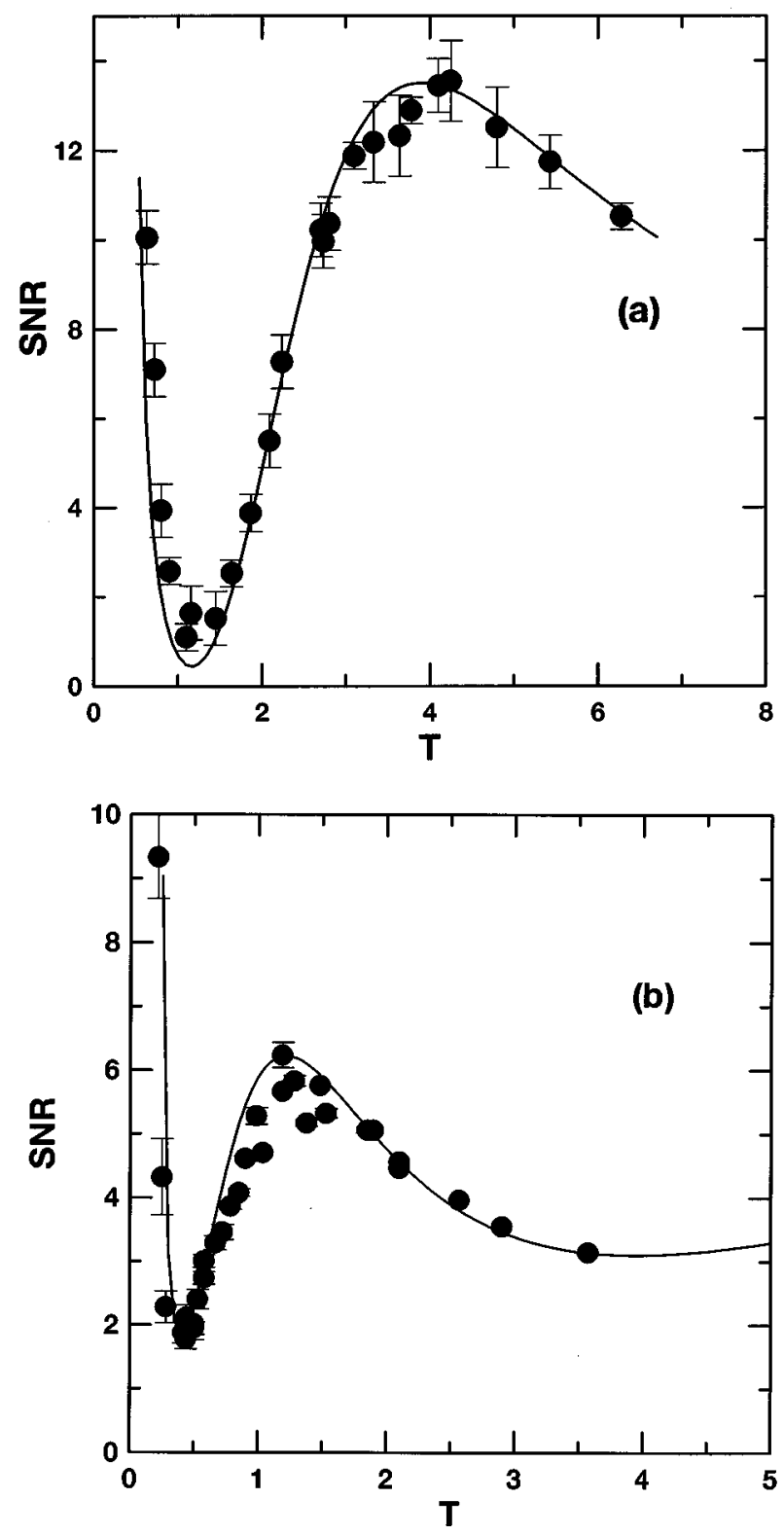

FIG. 8. The dependence of the signal-to-noise ratio on noise intensity for (a) the single-well SQUID potential of Fig. 3(a), with $\Omega=0.62$ and $A=0.016$; and (b) the multiwell SQUID potential of Fig. 3(b), with $\Omega=0.39$ and $A=0.005$. The results of the electronic experiments and numerical calculations are shown, respectively, by the circles and the solid lines.

lators quite generally displays a resonantlike behavior, in contrast to the behavior of the SNR in conventional systems. At the same time, the width of each resonance is generally much larger than the band of frequencies within which conventional SR is manifested in overdamped SQUIDs.

Finally, in this section, we comment that the extremal frequencies can be varied over a wide range by adjustment of the parameters $B$ and $q_{\mathrm{dc}}$, i.e., by changing the inductance or critical supercurrent and the external magnetic field.

\section{SUMMARY AND CONCLUSIONS}

We have demonstrated above, both theoretically and experimentally, that a substantial increase of SNR in an under-
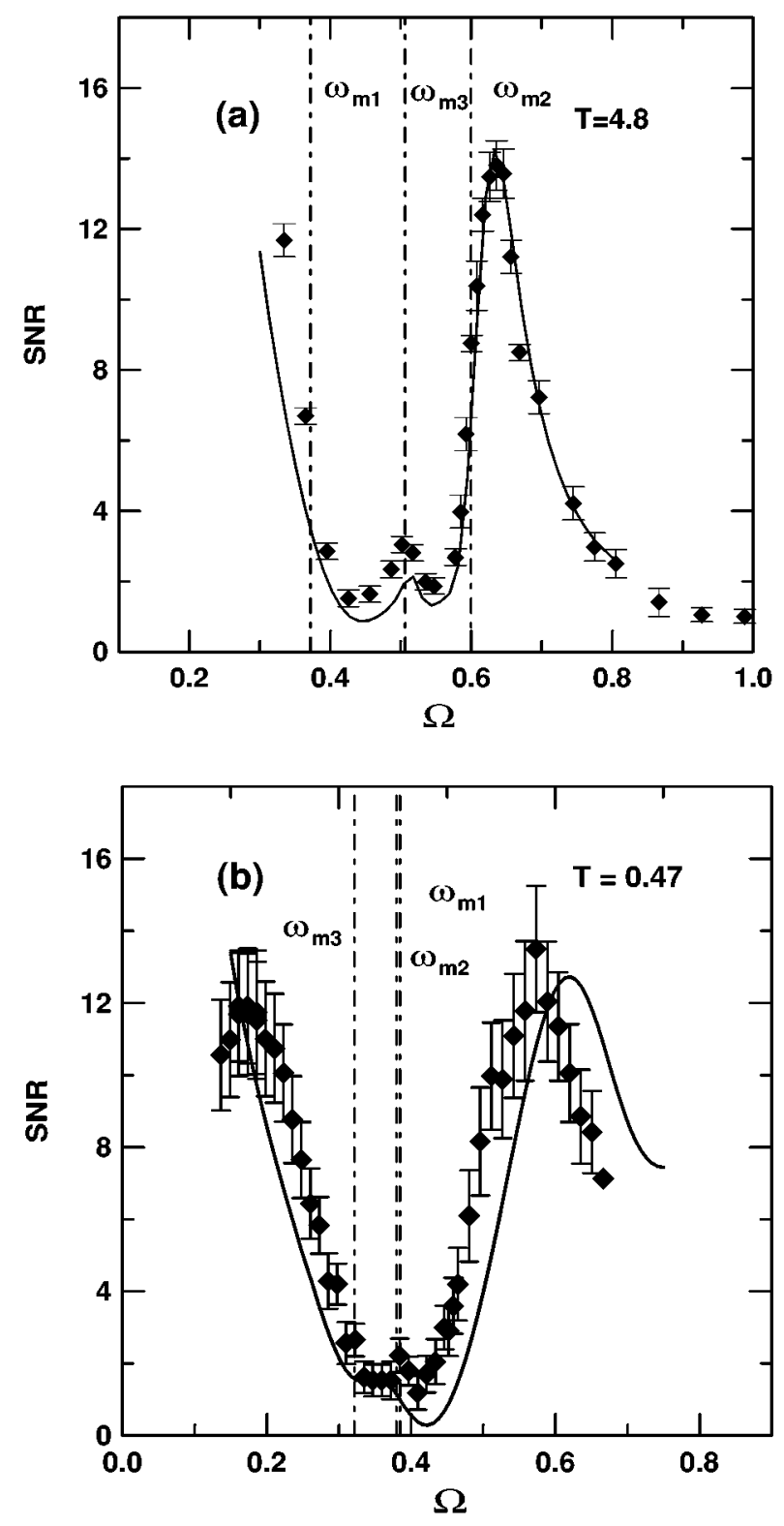

FIG. 9. The experimental (data points) and numerically calculated (solid line) frequency dependence of the SNR at fixed noise intensity for (a) the monostable potential and (b) the multiwell potential. Dash-dotted lines mark the positions of the relevant extrema of $\omega(E)$ (see Fig. 4).

damped superconducting loop with a Josephson junction can be achieved by the addition of noise. This ZDSR phenomenon arises in SQUIDs because of extrema in the dependence of their frequencies of eigenoscillation on energy which give rise, in turn, to characteristic asymmetric zerodispersion peaks in the fluctuation spectrum whose magnitude sharply increases with temperature. Thus the increase of SNR can be very large (infinite, in the limit of zero dissipation). The SNR also exhibits a rapid increase with changing frequency in the direction of the steepest decrease of the associated ZDP; and this increase usually occurs over a broader range of frequencies than the width of the ZDP itself. The evolution of fluctuation spectra with increasing noise intensity takes place through sequential activation of the zero-dispersion peaks corresponding to different extrema 
of $\omega(E)$ and exhibits an increasing magnitude for each successive peak. Thus, the SNR increase can take place over a wide range of signal frequencies and SQUID parameters; the SQUID can be designed to suit a given signal frequency through adjustment of its inductance or critical supercurrent, and also tuned by varying the external magnetic field.

Given that a superconductive loop with a Josephson junction constitutes the central element of a radio-frequency SQUID and of a variety of other SQUID-based devices, it is clear that ZDSR could in principle be used to improve the SNR at the output in many such applications. It should be noted also that an investigation of ZDSR in arrays of underdamped SQUIDs could be of interest, given that the coupling has been shown [34] to enhance the SNR for conventional SR in arrays of overdamped SQUIDs.

Finally, note that in the course of the work we have also obtained, as byproducts, two useful results: (a) a universal asymptotic function (12) describing the SNR in any ZD system, valid for the limit of a small dissipation; and (b) a convenient method for the numerical computation of fluctuation spectra in underdamped bistable and multistable systems, as a generalization of the method developed earlier for the monostable case [21].

\section{ACKNOWLEDGMENTS}

The work was supported by the Engineering and Physical Sciences Research Council (UK), by the Royal Society of London, by the International Science Foundation under Grant Nos. UBY2/000 and N623/000, and by the Russian Foundation for Basic Research.
[1] M. I. Dykman, D. G. Luchnsky, R. Mannella, P. V. E. McClintock, N. D. Stein, and N. G. Stocks, Nuovo Cimento D 17, 661 (1995); and other papers in this special issue devoted to stochastic resonance and related phenomena.

[2] A. R. Bulsara and L. Gamaitoni, Phys. Today 49 (3), 39 (1996)

[3] R. Benzi, A. Sutera, and A. Vulpiani, J. Phys. A 14, L453 (1981).

[4] C. Nicolis, Tellus 34, 1 (1982).

[5] B. McNamara, K. Wiesenfeld, and R. Roy, Phys. Rev. Lett. 60, 2626 (1988).

[6] B. McNamara and K. Wiesenfeld, Phys. Rev. A 39, 4854 (1989).

[7] M. I. Dykman, R. Mannella, P. V. E. McClintock, and N. G. Stocks, Phys. Rev. Lett. 65, 2606 (1990).

[8] N. G. Stocks, N. D. Stein, S. M. Soskin, and P. V. E. McClintock, J. Phys. A 25, L1119 (1992).

[9] N. G. Stocks, N. D. Stein, and P. V. E. McClintock, J. Phys. A 26, L385 (1993).

[10] The authors of a recent paper [11] have claimed the first report of the prediction and discovery of SR in monostable systems. We point out that the phenomenon was originally identified in [8,9]; its observation in a SQUID model was reported in [16].

[11] J. G. M. Vilar and J. M. Rubi, Phys. Rev. Lett. 77, 2863 (1996).

[12] S. M. Soskin, Physica A 155, 401 (1989).

[13] S. M. Soskin, Physica A 180, 386 (1992).

[14] N. G. Stocks, P. V. E. McClintock, and S. M. Soskin, Europhys. Lett. 21, 395 (1993).

[15] P. V. E. McClintock, S. M. Soskin, N. D. Stein, and N. G. Stocks, Phys. Rev. E 48, 147 (1993).

[16] I. Kh. Kaufman, D. G. Luchinsky, P. V. E. McClintock, S. M. Soskin, and N. D. Stein, Phys. Lett. A 220, 219 (1996).

[17] R. Barone and G. Paterno, Physics and Applications of the Josephson Effect (Wiley, New York, 1982).

[18] A. D. Hibbs, A. L. Singsaas, E. W. Jacobs, A. R. Bulsara, J. J. Bekkedahl, and F. Moss, J. Appl. Phys. 77, 2582 (1995).

[19] L. D. Landau and E. M. Lifshitz, Mechanics (Pergamon, London, 1976).

[20] Strictly, $R\left(\left[\Omega-\omega_{m}\right] / \Delta \omega\right)$ also depends on $T$ because $\Delta \omega$ $\propto T^{1 / 2}$. This dependence is much weaker, however, than the activation-law type dependence of $C_{\text {scale }}$ on $T$, and therefore has only a minor influence.

[21] M. I. Dykman, R. Mannella, P. V. E. McClintock, S. M. Soskin, and N. G. Stocks, Phys. Rev. A 42, 7041 (1990).

[22] H. Risken, The Fokker-Planck Equation, 2nd ed. (SpringerVerlag, Berlin, 1992).

[23] This procedure is equivalent to neglecting the nonresonant terms in the original FPE (13), written in an energy-angle representation in accordance with an averaging method [24] (see also $[12,13])$.

[24] N. N. Bogolyubov and Yu. A. Mitropolsky, Asymptotic Methods in the Theory of Nonlinear Oscillators (Gordon and Breach, New York, 1961).

[25] The case $n=0$ requires separate analysis and will be considered elsewhere. Our primary goals in the present paper are the calculations of the susceptibility and the SNR related to a zerodispersion peak. An evaluation of $W_{0}^{(j)}$ which determines the zero-frequency peak [26] is not necessary for present purposes.

[26] M. I. Dykman, R. Mannella, P. V. E. McClintock, S. M. Soskin, and N. G. Stocks, Phys. Rev. A 43, 1701 (1991).

[27] L. Fronzoni, in Noise in Nonlinear Dynamical Systems, edited by F. Moss and P.V.E. McClintock (Cambridge University Press, Cambridge, England, 1989), Vol. 3, p. 222.

[28] P. V. E. McClintock and F. Moss, in Ref. [27], p. 243.

[29] The AD534LD analog multiplier integrated circuit from Analog Devices, Box 9106, Norwood, MA 02062, USA.

[30] The AD639BD trigonometric integrated circuit from Analog Devices, Box 9106, Norwood, MA 02062, USA.

[31] J. Casedemunt, J. I. Jiménez-Acquino, J. M. Sancho, C. J. Lambert, R. Mannella, P. Martano, P. V. E. McClintock, and N. G. Stocks, Phys. Rev. A 40, 5915 (1989).

[32] From Microstar Laboratories Inc., 2265 116th Avenue NE, Bellevue, WA 98004, USA.

[33] The numerically calculated frequency dependence of SNR does not reproduce the left wing of the left peak of the experimentally measured dependence because it does not take into account the zero-frequency peak [25]. In other frequency ranges the agreement can be considered as quite satisfactory, especially given the exponentially sharp sensitivity of the results to the circuit parameters and noise intensity.

[34] A. Bulsara and G. Schmera, Phys. Rev. E 47, 3734 (1993). 